Almério de Castro Gomes'

Delsio Natal'

Marcia Bicudo de Paula'

Paulo Roberto Urbinatti'

Luís Filipe Mucci'

Marisa Dantas Bitencourt"

\section{Riqueza e abundância de Culicidae (Diptera) em área impactada, Mato Grosso do Sul, Brasil}

\section{Richness and abundance of Culicidae (Diptera) in an area impacted, Mato Grosso do Sul, Midwestern Brazil}

\section{RESUMO}

O represamento do Rio Pananá para construção da hidrelétrica de Porto Primavera, entre os Estados do Mato Grosso do Sul e São Paulo, alterou as relações ecológicas na região. $\mathrm{O}$ objetivo do estudo foi descrever a fauna de culicídeos potencialmente vetores nesse reservatório, a $2 \mathrm{~km}$ da margem direita, em Bataguassu, Mato Grosso do Sul, na fase anterior à inundação. Os culicídeos foram capturados em ambientes distintos mensalmente, de julho de 1997 a novembro de 1999. Foram calculados índices de riqueza e abundância. Obtiveram-se 16.553 exemplares adultos e 1.795 imaturos, com riqueza de 86 e 44 espécies, respectivamente. A fauna culicídea da área de estudo apresentava relativa riqueza, com espécies de valor epidemiológico, como o Anopheles darlingi, além de outras com potencial na veiculação de arbovírus.

DESCRITORES: Culicidae. Ecologia. Represas. Impacto ambiental. Saúde ambiental.

\section{ABSTRACT}

The damming of Paraná River for the construction of Porto Primavera Hydroelectric Power Plant, between the states of Mato Grosso do Sul, Midwestern Brazil and São Paulo, Southeastern Brazil, has changed the ecological relationships in the area. The objective of the study was to describe the fauna that can be potential vectors in this reservoir, $2 \mathrm{~km}$ away from its right bank, in Bataguassu, Mato Grosso do Sul, before flooding. Mosquitoes were monthly captured in different environments from July 1997 to November 1999. During this period 16,553 adult specimens and 1,795 immature forms were collected with richness of 86 different species of adults and 44 different species of immature forms. The fauna presented a richness that included species of epidemiological importance, as Anopheles darlingi and other mosquitoes with potential for arbovirus transmission.

KEY WORDS: Culicidae. Ecology. Dams. Environmental impact. Environmental health. 


\section{INTRODUÇÃO}

Em decorrência da exploração de madeira ou pela expansão da agropecuária, extensas áreas de vegetação natural vêm sendo substituídas por grandes espaços abertos, o que repercute na alteração dos hábitats de várias espécies da fauna e flora. Os mosquitos são sensíveis às mudanças ambientais, daí a necessidade do conhecimento das respostas adaptativas das espécies, induzidas pelas modificações antrópicas e seus reflexos na composição específica e abundância daquelas que sobreviveram.

A utilização dos recursos hídricos, por meio de represamento de rios, é outra forma que interfere nas comunidades biológicas em decorrência da transformação de ambientes lóticos em lênticos. Esses impactos afetam as imediações, demarcando contornos comumente reconhecidos como "área de influência".

A pressão antrópica vigente e a proximidade da área de alagamento da futura usina hidrelétrica Porto Primavera tornaram necessária a realização de um estudo da fauna de culicídeos. Tal estudo de avaliação da construção da hidrelétrica na expansão da abundância de vetores de interesse taxonômico em saúde pública possibilitará a descrição da reorganização da nova comunidade aquática da área.

No antigo Estado de Mato Grosso a fauna catalogada era restrita a alguns espécimes de Culicidae. ${ }^{5} \mathrm{O}$ objetivo do presente trabalho foi descrever, em seus aspectos de riqueza e abundância, a fauna Culicidae em período imediatamente anterior à inundação artificial.

\section{MÉTODOS}

A área estudada é composta por terrenos planos entremeados por resíduos de matas, brejos, alagadiços, pastagens e habitações humanas, pertencentes à Fazenda Santa Cruz (S21 $\left.{ }^{\circ} 44^{\prime} 31^{\prime \prime} / \mathrm{W} 52^{\circ} 16^{\prime} 03^{\prime \prime}\right)$, município de Bataguassu, Estado de Mato Grosso do Sul. Esta localidade está a cerca de $2 \mathrm{~km}$ da margem direita do futuro reservatório, quando na sua cota máxima de inundação. A região caracteriza-se por possuir uma rede de drenagem com amplos interflúvios que favorece acúmulo de águas pluviais nas áreas rebaixadas, com potencial para a proliferação de mosquitos. Os culicídeos foram capturados mensalmente no período de julho de 1997 a novembro de 1999. Foram explorados ambientes de capoeira, campo-aberto, brejo-aberto, mata-solo, mata-copa, mata-margem, mata, intradomicílio e peridomicílio.

Foram utilizadas várias técnicas e horários de capturas: aspiração de 15 min pela manhã; armadilha de Shannon (AScv) e a técnica atrativa humana (TAHcv) e armadilha do tipo CDC no crepúsculo vespertino, entre $17 \mathrm{~h}$ e $21 \mathrm{~h}$; a técnica atrativa humana durante 25 horas (TAH25h, que corresponde a $24 \mathrm{~h}$ e mais uma hora do efeito de intrusão). Todas as capturas foram realizadas com equipamentos de proteção pessoal para evitar eventuais picadas de mosquitos. Os mosquitos imaturos, larvas e pupas, foram capturados com conchas de alumínio (500ml), no mesmo período de captura dos adultos. A água era examinada até seu esgotamento nos pequenos criadouros no solo, em ocos de árvore e em recipientes domésticos.

A riqueza, traduzida na composição da fauna, foi estimada pela contagem do número de espécies ou táxons. Para o cálculo da abundância de mosquitos adultos recorreu-se ao índice de abundância de espécies padronizado (IAEP), proposto por Roberts \& $\mathrm{Hsi}^{4}$ (1979).

A identificação de gêneros e espécies seguiu Reinert. ${ }^{3}$

\section{RESULTADOS}

Foram capturados 16.553 adultos de culicídeos, distribuídos em dez gêneros com riqueza de 86 espécies, com registros sobrepostos entre as técnicas: aspiração (61 espécies), CDC (34), AScv (64), TAHcv (42), efeito intrusão (33) e TAH24h (48). Na captura de imaturos, 1.795 exemplares corresponderam a sete gêneros e riqueza de 44 espécies. Todas as espécies capturadas estão descritas na Tabela. O ano de 1998 foi o mais produtivo em diversidade e riqueza.

Entre os adultos, as espécies mais freqüentes nas coletas foram Anopheles triannulatus, Aedes scapularis e Coquillettidia hermanoi, perfazendo $48,5 \%$ do total. Entre os imaturos, destacaram-se An. albitarsis, Cx. (Cux.) complexo Coronator e Psorophora (Gra.) confinnis, totalizando $48,5 \%$.

As espécies predominantes na área estudada, para os anos de 1998 e 1999, estão indicadas pelos índices de abundância, por meio das técnicas TAHcv e AScv. An. triannulatus predominou, exceto no ano de 1999 pela TAHcv, quando foi suplantada por Ae. scapularis. An. darlingi esteve ausente em 1998, mas em 1999 ocupou $14^{\mathrm{a}}$ e quarta posições nas técnicas TAHcv e AScv, respectivamente.

As espécies mais abundantes coletadas somente pela TAH24h, no período de fevereiro de 1998 a janeiro de 1999, foram An. triannulatus, Ae. scapularis e Ae. serratus, dentre o total encontrado de 38 posições de abundância. 
Tabela. Distribuição das espécies de Culicidae. Bataguassu, MS, julho de 1997 a novembro de 1999.

\begin{tabular}{|c|c|c|c|c|c|}
\hline Gênero / Espécie & $\mathrm{N}$ & $\%$ & Gênero / Espécie (continuação) & $\mathrm{N}$ & $\%$ \\
\hline Anopheles triannulatus* & 3.699 & 20,16 & Psorophora (Grabhamia) spp.** & 15 & 0,08 \\
\hline Aedes scapularis* & 3.048 & 16,61 & Culex (Melanoconion) zeteki & 14 & 0,08 \\
\hline Coquillettidia hermanoi & 1.431 & 7,80 & Psorophora ferox & 14 & 0,08 \\
\hline Culex (Melanoconion) seção Melanoconion* & 930 & 5,07 & Culex (Culex) chidesteri* & 13 & 0,07 \\
\hline Anopheles albitarsis s.l.* & 874 & 4,76 & Aedes serratus/nubilus/aenigmaticus & 11 & 0,06 \\
\hline Aedes serratus & 781 & 4,26 & Sabethes glaucodaemon & 11 & 0,06 \\
\hline Anopheles braziliensis* & 701 & 3,82 & Culex (Melanoconion) evansae* & 10 & 0,05 \\
\hline Culex (Melanoconion) grupo Atratus* & 690 & 3,76 & Culex (Melanoconion) idottus* & 10 & 0,05 \\
\hline Culex (Culex) spp.* & 681 & 3,71 & Mansonia amazonensis & 10 & 0,05 \\
\hline Psorophora confinnins* & 607 & 3,31 & Psorophora ciliata* & 10 & 0,05 \\
\hline Culex (Culex) complexo Coronator* & 479 & 2,61 & Anopheles evansae & 9 & 0,05 \\
\hline Culex (Melanoconion) aureonotatus* & 395 & 2,15 & Anopheles rondoni & 9 & 0,05 \\
\hline Mansonia titillans & 395 & 2,15 & Uranotaenia hystera & 9 & 0,05 \\
\hline Culex (Melanoconion) theobaldi* & 385 & 2,10 & Uranotaenia spp.* & 9 & 0,05 \\
\hline Uranotaenia pulcherrima* & 363 & 1,98 & Culex (Melanoconion) pilosus $*$ & 8 & 0,04 \\
\hline Culex (Melanoconion) dunni* & 345 & 1,88 & Aedes aenigmaticus & 7 & 0,04 \\
\hline Aedeomyia squamipennis* & 245 & 1,34 & Aedes albopictus* & 7 & 0,04 \\
\hline Anopheles peryassui & 231 & 1,26 & Coquillettidia venezuelensis* & 7 & 0,04 \\
\hline Coquillettidia nigricans & 147 & 0,80 & Anopheles (Nyssorhynchus) spp.* & 6 & 0,03 \\
\hline Culex (Melanoconion) vaxus* & 146 & 0,80 & Anopheles strodei & 6 & 0,03 \\
\hline Culex (Culex) quinquefasciatus* & 130 & 0,71 & Psorophora cingulata & 6 & 0,03 \\
\hline Anopheles deaneorum & 117 & 0,64 & Aedes sp. & 5 & 0,03 \\
\hline Anopheles darlingi* & 109 & 0,59 & Aedes fulvus & 4 & 0,02 \\
\hline Anopheles galvaoi* & 98 & 0,53 & Coquillettidia chrysonotum/albifera & 4 & 0,02 \\
\hline Uranotaenia geometrica & 97 & 0,53 & Culex (Melanoconion) rorotaensis & 4 & 0,02 \\
\hline Culex (Melanoconion) bastagarius* & 96 & 0,52 & Mansonia humeralis & 4 & 0,02 \\
\hline Coquillettidia juxtamansonia & 82 & 0,45 & Aedes nubilus & 3 & 0,02 \\
\hline Coquillettidia shannoni & 74 & 0,40 & Aedes oligopistus & 3 & 0,02 \\
\hline Coquillettidia albicosta* & 72 & 0,39 & Anopheles albitarsis/deaneorum & 3 & 0,02 \\
\hline Culex (Culex) bidens* & 71 & 0,39 & Anopheles oswaldoi & 3 & 0,02 \\
\hline Culex (Culex) coronator* & 71 & 0,39 & Mansonia wilsoni & 3 & 0,02 \\
\hline Psorophora albigenu & 59 & 0,32 & Aedes hastatus & 2 & 0,01 \\
\hline Culex (Culex) mollis & 57 & 0,31 & Culex (Melanoconion) commevynensis & 2 & 0,01 \\
\hline Culex (Melanoconion) spp. & 48 & 0,26 & Culex (Melanoconion) delpontei & 2 & 0,01 \\
\hline Uranotaenia lowii ${ }^{*}$ & 36 & 0,20 & Culex (Melanoconion) grupo Intrincatus & 2 & 0,01 \\
\hline Anopheles evansae/galvaoi & 35 & 0,19 & Culex (Culex) camposi & 1 & 0,01 \\
\hline Culex (Culex) declarator* & 34 & 0,19 & Culex (Culex) nigripalpus & 1 & 0,01 \\
\hline Culex (Melanoconion) ocossa & 32 & 0,17 & Culex (Melanoconion) andricus & 1 & 0,01 \\
\hline Culex (Melanoconion) pavlovskyi & 29 & 0,16 & Culex (Melanoconion) clarki & 1 & 0,01 \\
\hline Culex (Melanoconion) ribeirensis & 28 & 0,15 & Culex (Melanoconion) contei & 1 & 0,01 \\
\hline Psorophora discrucians & 28 & 0,15 & Culex (Melanoconion) quasihibridus (próximo) & 1 & 0,01 \\
\hline Anopheles rondoni/strodei & 24 & 0,13 & Culex (Melanoconion) grupo Atratus morfoespécie $2^{* * *}$ & 1 & 0,01 \\
\hline Culex (Melanoconion) grupo Pilosus* & 23 & 0,13 & Limatus durhami & 1 & 0,01 \\
\hline Culex (Aedinus) amazonensis & 17 & 0,09 & Mansonia indubitans & 1 & 0,01 \\
\hline Culex (Culex) maxi* & 17 & 0,09 & Psorophora sp. & 1 & 0,01 \\
\hline Culex (Culex) saltanensis & 16 & 0,09 & Uranotaenia calosomata & 1 & 0,01 \\
\hline Culex (Melanoconion) rabelloi & 15 & 0,08 & Uranotaenia leucoptera & 1 & 0,01 \\
\hline Total & & & & 8.348 & 100,00 \\
\hline
\end{tabular}

* Adulto e larva

** Espécie não descrita

*** Kobayashi KM. Revisão das espécies do grupo Atratus de Culex (Melanoconion) (Diptera: Culicidae). [Dissertação de Mestrado]. São Paulo: Faculdade de Saúde Pública da USP; 1999. 


\section{DISCUSSÃO}

A fauna afetada pela intensa atividade antrópica é evidenciada pela presença marcante de Ae. scapularis, espécie indicadora de terrenos degradados. Da mesma forma a escassez de mosquitos do subgênero Melanoconion, cuja maioria das espécies são exclusivas de hábitats silvestres ${ }^{1}$ indicam o impacto ambiental. Mesmo diante do estresse ambiental, a fauna de culicídeos ainda guardava relativa riqueza, com potencial sinantrópico para espécies geradoras de incômodo ou veiculadoras de patógenos.

A análise do resultado do IAEP para as três modalidades de técnicas de coleta mostrou especificidade diferenciada. An. triannulatus predominou sobre as demais espécies, estimulada pela presença humana e luz das armadilhas.

Dentre os mosquitos que provocam incômodo, predominaram Cq. hermanoi e Mansonia titillans. De modo geral, os gêneros Mansonia e Coquillettidia apresentaram pouca abundância, mesmo diante da presença massiva de macrófitas nas lagoas locais. Entre as espécies sinantrópicas, coletaram-se Ae. albopictus e $C x$. quinquefasciatus, porém, pouco abundantes. As demais espécies são de reduzida ou nenhuma atividade antropofílica.

A ausência de An. darlingi em 1998 e sua presença em 1999, tanto na TAHcv (14 ${ }^{\mathrm{a}}$ posição) como na AScv (quarta posição) não pode ser interpretada como

\section{REFERÊNCIAS}

1. Forattini OP, Gomes AC, Kakitani I, Marucci D. Observações sobre domiciliação de mosquitos Culex (Melanoconion), em ambiente com acentuadas modificações antrópicas. Rev Saude Publica 1991;25(4):257-66.

2. Nasci RS. A light weight battery-powered aspirator for collecting resting mosquitoes in the field. Mosq News. 1981;41:808-11.

3. Reinert JF. Mosquito generic and subgeneric efeito da construção da barragem. Isso pela distância do canteiro de obras e porque, nesse período, a região ainda não havia sofrido qualquer interferência relativa à hidrelétrica. Portanto, fica sem explicação o surgimento súbito dessa espécie e com abundância relativamente alta.

Como visto, a dinâmica interpopulacional da fauna apresenta-se complexa, pois transcorre sob uma variabilidade influenciada ora pela escolha do estímulo usado pela técnica, ora por fatores do ambiente. Isto ficou mais evidente quando a coleção de água era mais volumosa, permanente ou temporária. A chuva parecia ser fator regulador dos ciclos reprodutivos dos mosquitos, ficando difícil definir os limites mínimos de água exigidos por cada espécie. A explicação mais razoável é que a configuração da riqueza e abundância está no valor adaptativo do culicídeo, em etapas do processo de domiciliação. Nesse gradiente ecológico, a presença humana e de animais domésticos influenciaram na predominância das espécies, cuja dinâmica de relacionamento com humanos faz emergir as espécies de interesse em saúde pública.

Conclui-se que, não obstante a devastação ocorrida, a região estudada era, naquele momento, detentora de fauna rica em culicídeos com presença de importantes espécies de valor epidemiológico. Uma delas é o $A n$. darlingi, além de outras com potencial na veiculação de arbovírus. Próximas comparações faunísticas ressaltarão os efeitos específicos do impacto gerado pela Represa Porto Primavera.

abbreviations (Diptera: Culicidae). Mosq Syst. 1975; 7: 105-10

4. Roberts DR, Hsi BP. An index of species abundance for use with mosquito surveillance data. Environ Entomol. 1979;8:1007-13.

5. Xavier SH, Mattos SS. Distribuição geográfica dos culicíneos no Brasil (Diptera, Culicidae). II Estado de Mato Grosso. Rev Bras Malariol Doenças Trop. 1970;22(2/4):441-60.

Financiado pela Fundação de Amparo a Pesquisa do Estado de São Paulo (Fapesp - Processo n. ${ }^{\circ}$ 96/10014-1) e Companhia Energética de São Paulo (CESP). 